\title{
Fabrication of Endothelial Cell-Laden Carrageenan Microfibers for 2Microvascularized Bone Tissue Engineering Applications
}

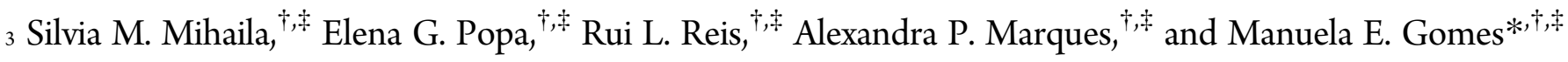 \\ $4{ }^{\dagger}$ 3B’s Research Group - Biomaterials, Biodegradables and Biomimetics, University of Minho, Headquarters of the European Institute \\ 5 of Excellence on Tissue Engineering and Regenerative Medicine, AvePark, 4806-909 Taipas, Guimarães, Portugal \\ $6{ }^{\star}$ ICVS/3B’s - PT Government Associate Laboratory, 4710-243 Braga, Guimarães, Portugal
}

\section{S Supporting Information}

8 ABSTRACT: Recent achievements in the area of tissue engineering (TE) have enabled the development of threedimensional (3D) cell-laden hydrogels as in vitro platforms that closely mimic the $3 \mathrm{D}$ scenario found in native tissues. These platforms are extensively used to evaluate cellular behavior, cell-cell interactions, and tissue-like formation in highly defined settings. In this study, we propose a scalable and flexible 3D system based on microsized hydrogel fibers that might be used as building blocks for the establishment of 3D hydrogel constructs for vascularized bone TE applications. For this purpose, chitosan $(\mathrm{CHT})$ coated $\kappa$-carrageenan $(\kappa$-CA) microfibers were developed using a two-step procedure

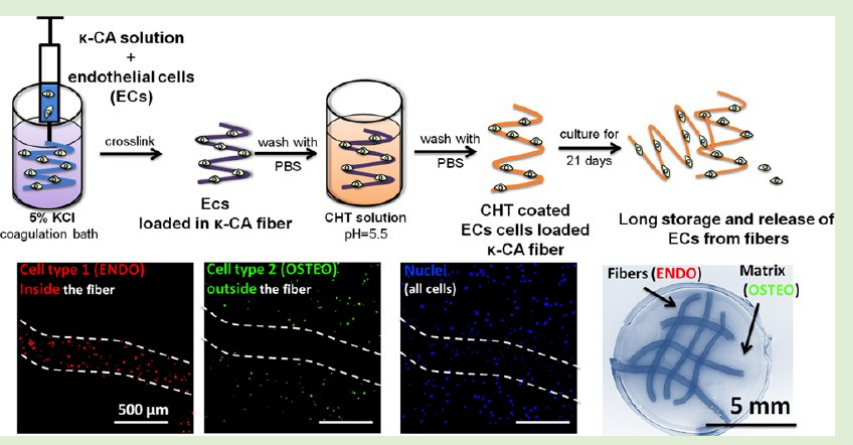
involving ionotropic gelation (for the fiber formation) of $\kappa$-CA and its polyelectrolyte complexation with $\mathrm{CHT}$ (for the enhancement of fiber stability). The performance of the obtained fibers was assessed regarding their swelling and stability profiles, as well as their ability to carry and, subsequently, promote the outward release of microvascular-like endothelial cells (ECs), without compromising their viability and phenotype. Finally, the possibility of assembling and integrating these cell-laden fibers within a 3D hydrogel matrix containing osteoblast-like cells was evaluated. Overall, the obtained results demonstrate the suitability of the microsized $\kappa$-CA fibers to carry and deliver phenotypically apt microvascular-like ECs. Furthermore, it is shown that it is possible to assemble these cell-laden microsized fibers into $3 \mathrm{D}$ heterotypic hydrogels constructs. This in vitro $3 \mathrm{D}$ platform provides a versatile approach to investigate the interactions between multiple cell types in controlled settings, which may open up novel 3D in vitro culture techniques to better mimic the complexity of tissues.

\section{INTRODUCTION}

29 In the past decade, tissue engineering (TE) has emerged as a 30 multidisciplinary field at the interface of medicine, biology, and 31 engineering, aiming at fabricating tissue-like biological con32 structs. ${ }^{1}$ However, the lack of a vasculature that can sustain the 33 nutrients and oxygen demands within the tissue-engineered 34 construct is a major limiting factor in creating thick artificial 35 tissues. ${ }^{2}$ Thus, developing vessel-like networks based on 36 endothelial cells (ECs), as integrated templates within TE 37 constructs, ${ }^{3}$ would be essential for creating real-size replicas of 38 tissues or organs. 4

39 Hydrogels have been proven to provide ideal mimics of cellular 40 matrices, as their hydrated state resembles that of native 41 extracellular matrix (ECM) ${ }^{6}$ and their high permeability to 42 oxygen, nutrients, and metabolites diffusion ${ }^{7}$ enables cell 43 encapsulation. Additionally, natural-origin hydrogels showed 44 high potential in the TE field, due to their chemistry and 45 properties similar to ECM. ${ }^{8}$ Several processing methodologies 46 such as prototyping/printing, ${ }^{9}$ microfluidics, ${ }^{10}$ and photo47 litography ${ }^{11}$ have been used to develop vessel-like architectures 48 based on ECs-loaded hydrogels that exhibit microsized features $49\left(\sim 50 \mu \mathrm{m}^{11}\right.$ to $\left.1 \mathrm{~mm}^{9}\right)$. However, these methods often require sophisticated equipment and elaborated experimental settings 50 and reagents that frequently raise concerns over their 51 cytotoxicity. ${ }^{12,13}$

Wet spinning of hydrogels is a very simple and straightforward 53 method, requiring minimal laboratorial utensils and short 54 processing time. The wet spinning of hydrogel fibers involves 55 the extrusion of a polymer solution through a needle into a 56 coagulation bath that triggers the cross-linking of the polymer 57 into a fiber-like shape. This methodology has been successfully 58 used to develop hydrogel fibers based on several natural-origin 59 polymers, such as alginate, ${ }^{14}$ collagen, ${ }^{15}$ gellan gum, ${ }^{16}$ chitosan, ${ }^{17} 60$ or a combination of carrageenan and alginate. ${ }^{18}$

Carrageenans (CAs) stand out, among other natural-origin 62 polymers, as potential candidates for TE applications, due to 63 their mild gelation properties and resemblance to glycosamino- 64 glycans (GAGs), main components of the ECM of biological 65 systems. CAs are highly sulfated linear polysaccharides with 66 alternating repeating 3,6-anhydro-D-galactose and $\beta$-D-galactose- 67

Received: January 9, 2014

Revised: June 18, 2014 
68 4-sulfated units, that provide structural support in several species 69 of marine red algae (class of Rhodophyceae). Due to the half70 ester sulfate moieties present on their backbone (their amount 71 determines the type of carrageenan that can be iota, lambda, or 72 kappa), CAs are strong anionic polymers. As a consequence, their 73 gelation occurs by ionic interactions with appropriate counter74 ions, such as $\mathrm{K}^{+}, \mathrm{Na}^{+}$, or $\mathrm{Ca}^{2+}$. Although these hydrophilic 75 polysaccharides have been widely used as emulsifiers, gelling, 76 thickening, or stabilizing agents in the food or pharmaceutical 77 industry, ${ }^{19}$ the intrinsic thixotropic behavior of $\kappa$-carrageenan $(\kappa$ $78 \mathrm{CA}$ ), in particular, has justified its exploitation as an injectable 79 matrix for the delivery of living cells ${ }^{18,20,21}$ and biomacromole80 cules. $^{22,23}$ However, $\kappa$-CA hydrogels exhibit high swelling ratios 81 and mechanical instability in physiological conditions ${ }^{24}$ which 82 has motivated the development of several approaches to increase 83 their stability, such as chemical modifications with photo-cross84 linkable moieties, ${ }^{25}$ blending with other biopolymers, ${ }^{18,26}$ 85 addition of nanocomposites to the polymer solution, ${ }^{23}$ and 86 formation of interpenetrating networks ${ }^{27}$ or polyelectrolyte 87 complexation (PEC) with polycations, such as chitosan $88(\mathrm{CHT}){ }^{28}$ Numerous studies have reported CHT-based PEC 89 systems with positive outcomes, in terms of stability as well as 90 cellular viability and behavior. ${ }^{29,30}$ Electrostatic interactions 91 between $\kappa$-CA and CHT have also been used for the 92 development of nanoparticles, ${ }^{31}$ beads, ${ }^{32}$ and layer-by-layer 93 systems. $^{33}$

94 The present work reports on the development of $\kappa$-CA 95 microfibers coated with CHT using a two-step-procedure, and 96 on their potential as cell carriers and building blocks of 3D 97 constructs for vascularized bone tissue engineering. Therefore, in 98 a first step, $\kappa$-CA fibers of various diameters were obtained by wet 99 spinning. In a second step, in order to reinforce the fibers and 100 enhance their stability in physiological relevant microenviron101 ment, $\kappa$-CA fibers were coated with $\mathrm{CHT}$, through the 102 electrostatic interaction between the polymers. The obtained 103 fibers were then loaded with microvascular-like ECs obtained 104 from the SSEA- $4^{+}$subpopulation of adipose tissue stromal 105 vascular fraction, as described in a previous study. ${ }^{34}$ The 106 phenotype of the ECs was evaluated prior to and after 107 entrapment within the fibers. Ultimately, envisioning a 108 heterotypic 3D bone TE construct with independent and 109 defined microarchitectures, we proposed an innovative 3D 110 buildup of ECs loaded fibers within a hydrogel matrix containing 111 osteoblast-like cells. In summary, this study showed that it is 112 possible to developed cell-laden $\kappa$-CA-based hydrogel fibers that 113 exhibit high stability in prolonged culture conditions. Fur114 thermore, these fibers could be stacked in a desired pattern and 115 successfully integrated into a $3 \mathrm{D}$ construct.

\section{MATERIALS AND METHODS}

116 2.1. Materials. $\kappa$-Carrageenan $(\kappa$-CA), potassium chloride $(\mathrm{KCl})$, 117 and $\beta$-glycerophosphate disodium salt hydrate $(\beta \mathrm{GP})$ were purchased 118 from Sigma, Germany. Reagent grade medium molecular weight 119 chitosan (CHT) (Sigma, Germany) with a 90\% degree of acetylation 120 was used. Prior to use, $\mathrm{CHT}$ was purified using a precipitation method. ${ }^{35}$ 121 All other reagents were used as received.

122 2.2. Development of CHT Coated $\kappa$-CA Fibers. 2.2.1. Production 123 of $\kappa$-CA Fibers through lonotropic Gelation. The $\kappa$-CA hydrogel fibers 124 were obtained by wet spinning technique, which consisted of the 125 extrusion of the polymer solution through a needle immersed in a 126 coagulation bath, as previously described elsewhere. ${ }^{18}$ Briefly, a $1.5 \%$ $127(\mathrm{wt} / \mathrm{v}) \kappa$-CA solution was prepared by dissolving the polymer in distilled 128 water at $50{ }^{\circ} \mathrm{C}$ and under constant stirring until complete dissolution 129 was achieved. Subsequently, the $\kappa$-CA solution was loaded into $5 \mathrm{~mL}$ syringes and $\kappa$-CA fibers with different diameters were obtained by 130 extruding the polymeric solution through needles with different gauges 131 $(18 \mathrm{G}$ to $27 \mathrm{G})$, directly into a $5 \%(\mathrm{wt} / \mathrm{v}) \mathrm{KCl}$ solution prepared in 132 distilled water. The presence of $\mathrm{K}^{+}$ions initiated the ionotropic gelation 133 by counterbalancing the negative charges of $\kappa$-CA. The fibers were 134 allowed to harden in the coagulation bath for about $10 \mathrm{~min}$, sufficient 135 time for them to retain the shape. Finally, the fibers were washed with 136 phosphate buffered saline (PBS) in order to remove the excess of salt. 137 Fibers obtained with needles of $25 \mathrm{G}$ and $27 \mathrm{G}$ were selected for all the 138 subsequent assays.

2.2.2. Optimization of the $\mathrm{pH}$ of the CHT Working Solution. 140 Chitosan dissolves in acid solutions, which limits its use in the presence 141 of living cells. Previous studies ${ }^{36}$ have shown that $\beta \mathrm{GP}$, a weak base, 142 increases the $\mathrm{pH}$ of $\mathrm{CHT}$ solutions, without jeopardizing its solubility. 143 Therefore, in order to establish optimal conditions for the incorporation 144 of cells, while enabling PEC, a curve of variation of $\mathrm{pH}$ with the addition 145 of $\beta \mathrm{GP}$ to the $\mathrm{CHT}$ solution was obtained. For this purpose, a CHT 146 solution was prepared by dissolving CHT in a $1 \%(\mathrm{v} / \mathrm{v})$ acetic acid 147 solution at a final concentration of $0.5 \%(\mathrm{wt} / \mathrm{v})$. In order to determine 148 the degree to which the addition of $\beta \mathrm{GP}$ affects the overall charge of 149 CHT solution, zeta potential measurements were performed using a 150 Malvern Zeta Sizer Nano ZS (Malvern Instruments, UK). Each sample 151 was diluted in ultrapure water at a concentration of $0.1 \%(\mathrm{wt} / \mathrm{v})$ and 152 analyzed at $25^{\circ} \mathrm{C}$ for $60 \mathrm{~s}$.

2.2.3. Coating of $\kappa$-CA Fibers through Polyelectrolyte Complex- 154 ation with $\mathrm{CHT}$. The $\kappa$-CA fibers, previously obtained by ionotropic 155 gelation, were immersed in the optimized CHT solution (0.5\% (wt/v) 156 and $\mathrm{pH}=5.5$ ) for $20 \mathrm{~min}$, followed by several washing steps with PBS to 157 remove the excess of CHT. The presence of the CHT coating was 158 evaluated by staining the fibers with Eosin Y (Sigma, Germany). Fibers 159 without coating were used as negative control.

2.3. Physico-Chemical Characterization of the Developed 161 Fibers. 2.3.1. Swelling Kinetics. The influence of CHT coating on the 162 swelling and stability of the developed $\kappa$-CA fibers was determined by 163 evaluating the fibers' water absorption kinetics and diameter variation 164 upon immersion in culture medium for up to 21 days. The culture 165 medium used for this purpose was Dulbecco's Modified Eagle Medium 166 (DMEM, Gibco, USA) supplemented with 10\% (v/v) HiFBS (Gibco, 167 USA) and $1 \%(\mathrm{v} / \mathrm{v}$ ) antibiotic/antimycotic (penicillin/streptomycin, 168 $100 \mathrm{U} / 100 \mu \mathrm{g} / \mathrm{mL}$, Gibco, UK). Envisioning the use of fibers for cell 169 encapsulation/culturing, the experimental parameters were set at 170 physiological temperature $\left(37^{\circ} \mathrm{C}\right)$ and humidified atmosphere with 171 $5 \%$ of $\mathrm{CO}_{2}$. Medium was replenished every 3-4 days. Fibers $(n=3) 172$ were retrieved at days 7,14, and 21, blotted with KimWipe paper to 173 remove the excess of liquid, and weighed $\left(M_{\mathrm{W}}\right)$. Sample's final weight 174 $\left(M_{\mathrm{DF}}\right)$ was determined upon lyophilization. The swelling kinetics was 175 defined as the ratio between the liquid uptake $\left(M_{\mathrm{W}}-M_{\mathrm{DF}}\right)$ and the final 176 dry mass of polymer $\left(M_{\mathrm{DF}}\right)$, according to eq 1 .

$$
\text { mass swelling ratio }=\left(M_{\mathrm{w}}-M_{\mathrm{DF}}\right) / M_{\mathrm{DF}} \times 100
$$

The final diameter of the hydrogel fibers was also measured applying 179 software-measuring tools (NIH ImageJ software, http://rsbweb.nih. 180 gov/ij/) to at least three micrographs of each sample.

2.3.2. Morphological and Chemical Characterization. Concom- 182 itant with the swelling behavior analysis, samples were retrieved for 183 surface morphological evaluation and elemental analysis under a 184 scanning electron microscope (SEM) coupled with energy-dispersive 185 $\mathrm{X}$-ray spectroscopy (EDX/EDS). Briefly, fibers were snap frozen in 186 liquid nitrogen, transferred to microcentrifuge tubes, and freeze-dried 187 overnight. The dried samples were carefully mounted on sample holders 188 using double-sided carbon tape. Before being analyzed by SEM (Nano- 189 SEM FEI Nova 200), the samples were gold sputter coated (Fisons 190 Instruments, sputter coater SC502, UK). Elemental analysis was carried 191 out with an energy dispersive spectrometer (EDAX-Pegasus X4M) on 192 the same samples used for SEM. All observations/image acquisitions 193 and measurements were made at an acceleration voltage of $15 \mathrm{kV}$.

2.4. Isolation and Endothelial Differentiation of SSEA- 195 $4^{+}$hASCs. Human lipoaspirate samples from healthy donors were 196 kindly provided by Hospital de Prelada (Porto, Portugal), under 197 previously established protocols and with the informed consent of the 198 
A

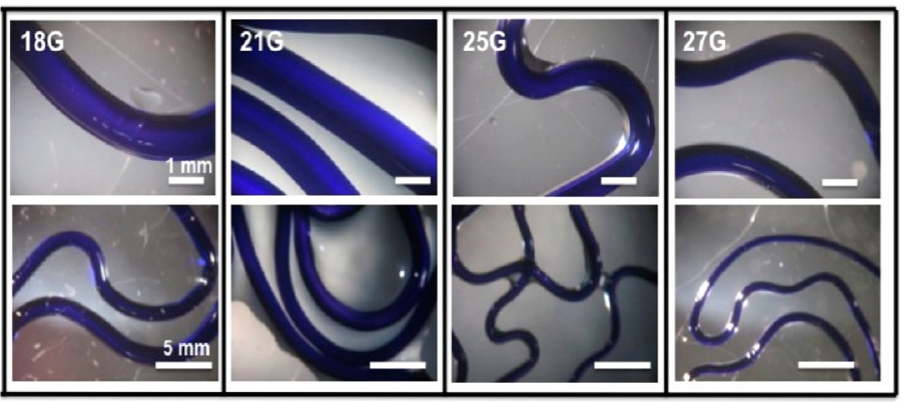

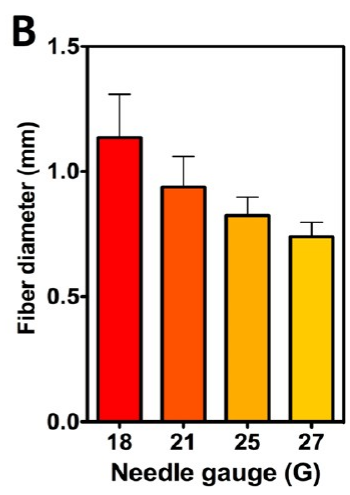

Figure 1. K-CA fibers formed by ionotropic gelation. Needles with different gauges, from $18 \mathrm{G}$ to $27 \mathrm{G}$, were used to form the hydrogel fibers. (A) Aspect of fibers using different needle gauges. Staining with methylene blue was performed for contrast purposes. (B) Depending on the needle gauge, fibers diameter spanned from $1.25 \mathrm{~mm}$ down to $0.5 \mathrm{~mm}$.

199 patients. The selection of SSEA-4 positive cells (SSEA- $4^{+}$hASCs) 200 residing within the stromal vascular fraction of the adipose tissue and 201 differentiation toward the endothelial lineage were performed according 202 to our previously published protocols. ${ }^{34}$ Briefly, the SSEA- $4^{+}$hASCs 203 were selected using immunomagnetic beads (Dynal M-450 Epoxy beads 204 from Dynal Biotech, Carlsbad, CA, USA) coated with SSEA-4 antibody 205 (Abcam, Cambridge, UK). Then, the selected SSEA-4 ${ }^{+}$hASCs cells were 206 differentiated toward endothelial lineage by culturing them for 2 weeks 207 in endothelial cell growth medium, EGM-2 MV (Lonza, Switzerland), 208 containing 5\% FBS and supplemental growth factors: hydrocortisone, 209 hFGF-B, ascorbic acid, gentamicin/amphotericin, VEGF, long R3-IGF2101 , hEGF, and heparin, at concentrations established by the 211 manufacturer.

212 2.5. SSEA-4 ${ }^{+}$hASCs-Derived Endothelial Cells Encapsulation 213 within $\kappa$-CA Fibers. The $\kappa$-CA, $\mathrm{CHT}$, and $\mathrm{KCl}$ solutions were 214 sterilized at $120^{\circ} \mathrm{C}$ for $30 \mathrm{~min}$. SSEA- $4^{+}$hASCs-derived ECs at passage 3 215 were trypsinized and centrifuged, and further suspended in the $\kappa$-CA 216 solution at a final cell density of $2 \times 10^{6}$ cells $/ \mathrm{mL}$. ECs loaded fibers 217 (with and without CHT coating) were produced as described above. 218 The cell-loaded fibers were then transferred to 24-well plates and 219 maintained in culture in EGM-2 MV for 21 days, at $37^{\circ} \mathrm{C}$ in a humidified 220 atmosphere with $5 \% \mathrm{CO}_{2}$. Culture medium was replenished every 3-4 221 days.

222 In order to assess the maintenance of the phenotype of the SSEA$2234^{+}$hASCs derived ECs at 21 days of culture cell-loaded fibers were 224 treated with $0.1 \%$ proteinase $\mathrm{K}$ (vWR, Portugal) in $1 \mathrm{mM}$ EDTA 225 (Sigma, Germany), $50 \mathrm{mM}$ TrisHCl (Sigma, Germany), and $1 \mathrm{mM}$ 226 iodoacetamide buffer (Sigma, Germany), for $1 \mathrm{~h}$ at $37{ }^{\circ} \mathrm{C}$, under 227 constant agitation, to release the cells from the fibers. The cellular pellet 228 recovered after centrifugation $(10 \mathrm{~min}, 400 \times \mathrm{g})$ was resuspended in 229 EGM-2 MV medium and plated into tissue culture flasks. Cells were 230 cultured until reaching confluence for further analysis, as described 231 below.

232 2.6. Characterization of the Constructs. 2.6.1. Microscopic 233 Analysis. Variations in the shape and diameter of the developed cell234 loaded fibers (with or without CHT coating), as well as the potential 235 outward migration of the cells to the culture well, were examined using a 236 stereomicroscope (Stemi 1000, Zeiss, Germany) along the time of 237 culture.

238 2.6.2. Live-Dead Assay. At preselected time culturing points (1, 7, 23914 , and 21 days), cell-loaded fibers were washed with PBS and incubated 240 with $4 \mu \mathrm{M}$ calcein-AM (Invitrogen, USA) for $40 \mathrm{~min}$ followed by $10 \mathrm{~min}$ 241 incubation with $1 \mu \mathrm{M}$ propidium iodide (PI, Invitrogen, USA). Samples $242(n=3)$ were then washed and fixed for $40 \mathrm{~min}$ in $10 \%$ formalin. After 243 fixation, samples were washed with PBS and cell nuclei were 244 counterstained for $20 \mathrm{~min}$ with 4,6-diamidino-2-phenyindole dilactate 245 (DAPI, Sigma, Germany) and then washed three times with PBS. 246 Representative fluorescent micrographs were acquired using the 247 Axioplan Imager Z1 fluorescence microscope (Zeiss, Germany) and 248 the AxioVision 4.8 software (Zeiss, Germany). The quantification of 249 cellular viability was measured by applying software-measuring tools
(NIH ImageJ software, http://rsbweb.nih.gov/ij/) to at least three 250 micrographs of each sample.

2.6.3. Flow Cytometry. SSEA-4 $4^{+}$hASCs-derived ECs, prior to and 252 after encapsulation, were retrieved from cell culture flasks using TrypLE 253 Express (Invitrogen, USA). $5 \times 10^{5}$ cells were incubated for $30 \mathrm{~min}$ at 4254 ${ }^{\circ} \mathrm{C}$ with the following markers: CD45-FITC, CD34-PE, CD73-PE, 255 CD31-APC (all from BD Pharmingen, USA), and CD105-FITC and 256 CD90-APC (eBiosciences, USA) at a concentration of $6 \mu \mathrm{g} / \mathrm{mL}$, as 257 recommended by the manufacturer. After washing with PBS, the cells 258 were resuspended in acquisition buffer (PBS containing 1\% form- 259 aldehyde and $0.1 \%$ sodium azide) and analyzed with a BD FACS-Calibur 260 flow cytometer (BD Biosciences, USA). A minimum of $2 \times 10^{5}$ events 261 was acquired and gated in a forward versus side-scatter dot plot with a 262 linear scale. Results were displayed in histogram plots created using 263 CellQuest software (BD Biosciences, USA). The number of positive 264 events for each cell-specific marker was expressed as a percentage of the 265 total cell number.

2.6.4. Matrigel Assay. SSEA- $4^{+}$hASCs-derived ECs, at passage 2, 267 were trypsinized and plated at a density of $3 \times 10^{4}$ cells/well in 48-well 268 plates coated with $64 \mu \mathrm{L}$ of Matrigel (BD Biosciences, USA). Cells were 269 incubated for $4 \mathrm{~h}$ at $37{ }^{\circ} \mathrm{C}$. One hour prior fixation, $4 \mu \mathrm{M}$ calcein-AM 270 (Invitrogen, USA) was added to the wells. Upon fixation, cell nuclei 271 were counterstained with DAPI for $10 \mathrm{~min}$, and then washed three times 272 with PBS. Three representative images were acquired under a 273 fluorescence microscope.

274

2.7. Assembling the $\kappa$-CA Fibers into 3D Hydrogel Discs. Cell- 275 laden fibers containing SSEA- $4^{+} \mathrm{hASCs}$-derived ECs labeled with a GFP 276 tag were transferred to a Petri dish and allowed to settle randomly. A 277 freshly prepared $\kappa$-CA solution containing osteogenic differentiated 278 SSEA- $4^{+}$hASCs (obtained according to a method described else- 279 where ${ }^{34}$ ) and labeled with a rhodamine tag was poured onto the fibers 280 until full coverage. The cross-linking of the $\kappa$-CA solution was achieved 281 with a $5 \%(\mathrm{wt} / \mathrm{v}) \mathrm{KCl}$ solution. Immediately after cross-linking, the 282 hydrogel disc containing the fibers was fixed with $10 \%$ formalin for 40283 min. After fixation, the cell nuclei were stained with DAPI for $20 \mathrm{~min}$ and 284 observed under a confocal laser scanning microscope (CLSM, Olympus, 285 Fluoview 1000). $Y Z$ and $X Y$ projections were performed in order to 286 evaluate the cellular distribution throughout the 3D structures. 287

2.8. Statistical Analysis. For the swelling ratio and fiber diameter 288 data, statistical analysis was performed using GraphPad Prism 5.00289 software (San Diego, USA). Statistical differences $(p<0.05)$ were 290 determined using one-way ANOVA, followed by a Tukey post test. 291

\section{RESULTS AND DISCUSSION}

3.1. Different Diameter $\kappa$-CA Fibers Formation through 292 lonotropic Gelation. Cell encapsulation techniques using 293 hydrogels enable the formation of 3D cell-culture models that 294 can potentially replicate tissue organization, which cannot be 295 achieved in conventional 2D cultures. ${ }^{37}$ Since the architecture 296 and chemical composition of hydrogels can be easily engineered, 297 


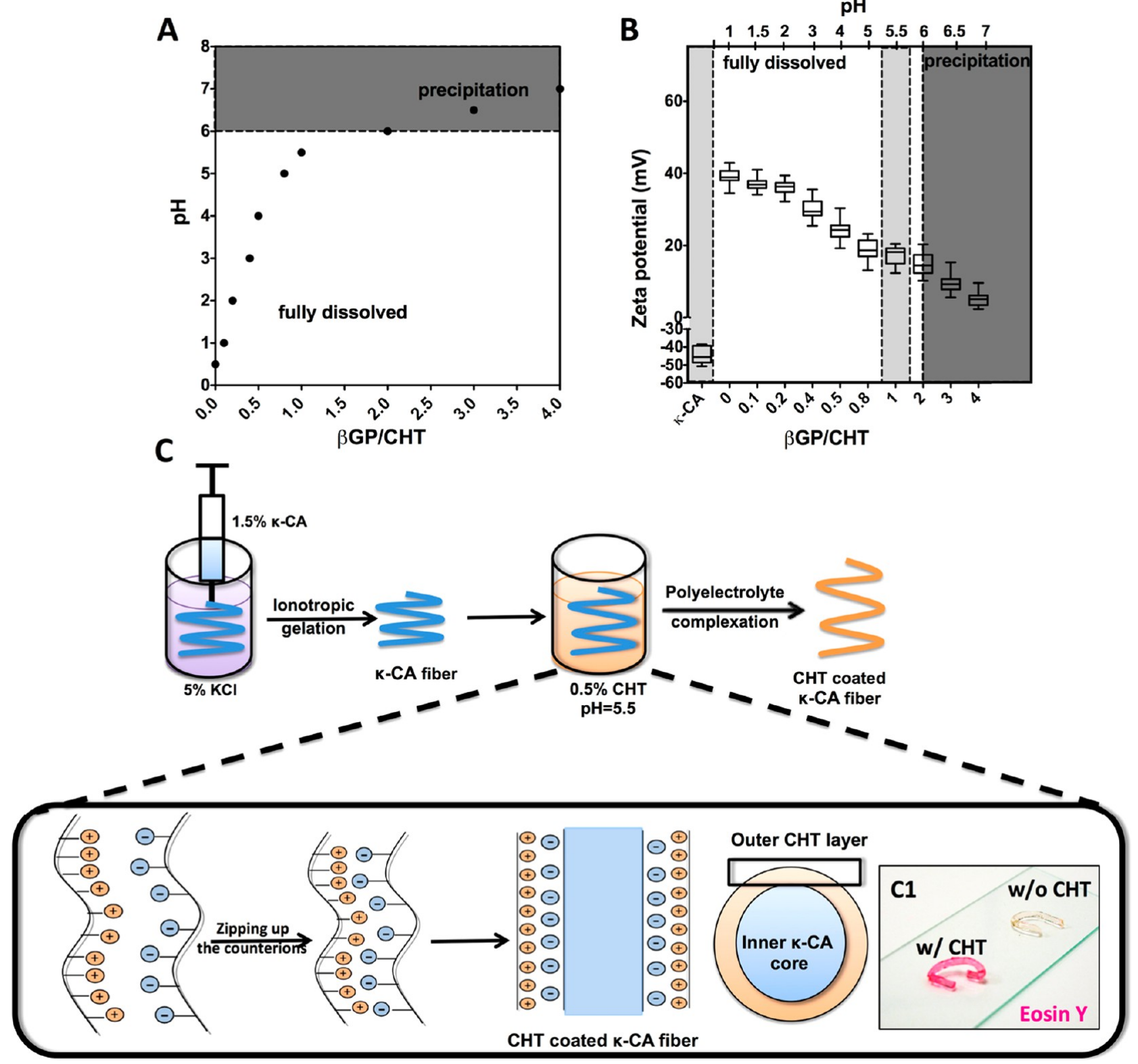

Figure 2. Production of $\mathrm{CHT}$ coated $\kappa$-CA hydrogel fibers. (A) Variation of the $\mathrm{pH}$ relative to the $\beta$-glycerophosphate $(\beta \mathrm{GP}) / \mathrm{chitosan}(\mathrm{CHT})$ ratio. With the addition of $\beta \mathrm{GP}$, the $\mathrm{CHT}$ solutions reach physiologically relevant conditions ( $\mathrm{pH} 5.5-6$ ). Above this point the precipitation of $\mathrm{CHT}$ occurs (dark gray area). (B) With the addition of $\beta \mathrm{GP}$, the zeta potential of CHT solution is dramatically affected. The zeta potential of $\kappa$-CA solutions, measured at $\mathrm{pH}=5.5$, was used as reference (approximately $-40 \mathrm{mV}$ ). Values reported correspond to $n=10$. (C) Schematics of the production of CHT coated $\kappa$-CA hydrogel fibers. The process involves the formation of fibers by ionotropic gelation in a $\mathrm{KCl}$ coagulation bath, followed by their immersion in an optimized CHT solution. (C1) The presence of the CHT coating was confirmed by staining the fibers with Eosin Y which specifically binds to CHT.

298 they can be used as tools and platforms to design capture/release 299 of cells in/from 3D tissue-engineered constructs. ${ }^{38}$

300 Thus, we aimed to develop hydrogel microfibers by making 301 use of a simple and straightforward procedure that enables cell 302 encapsulation in a controlled distribution pattern, without 303 jeopardizing their phenotype and functionality. Among the 304 natural origin polymers, such as alginate, ${ }^{14,13}$ collagen, ${ }^{15}$ gellan 305 gum, $^{16,39}$ or hyaluronic acid $^{40}$ that have been used to obtain 306 fibers through wet spinning technique, $\kappa$-CA stands out due to its 307 GAG-like features, reversible temperature and ionic gelation 308 properties and intrinsic sheer thinning behavior, particularly 309 thixotropic. This means that upon applied stress the organization 310 of $\kappa$-CA chains is disrupted, but it will reset once the deformation is removed. ${ }^{25}$ This property renders the use of $\kappa$-CA hydrogels as 311 injectable matrixes, as they can be easily extruded through narrow 312 needles without affecting the structure or the entrapped 313 biomacromolecules or cells. ${ }^{41,42}$ Taken together, the gelation 314 properties of $\kappa$-CA enable the formation of gels in different 315 shapes, including fibers, highlighting therefore the versatility of $\kappa$ - 316 CA processability. ${ }^{8}$

In the present study, the possibility of producing $\kappa$-CA fibers 318 with different diameters was first investigated. To obtain the fiber 319 shape, $\kappa$-CA was extruded directly into the coagulation bath, 320 where the cross-linking of the polymer solution occurred. 321 Variation of the diameter depended on the needle gauge used to 322 extrude the $\kappa$-CA solution into the coagulation bath (Figure 1A). $323 \mathrm{fl}$ 

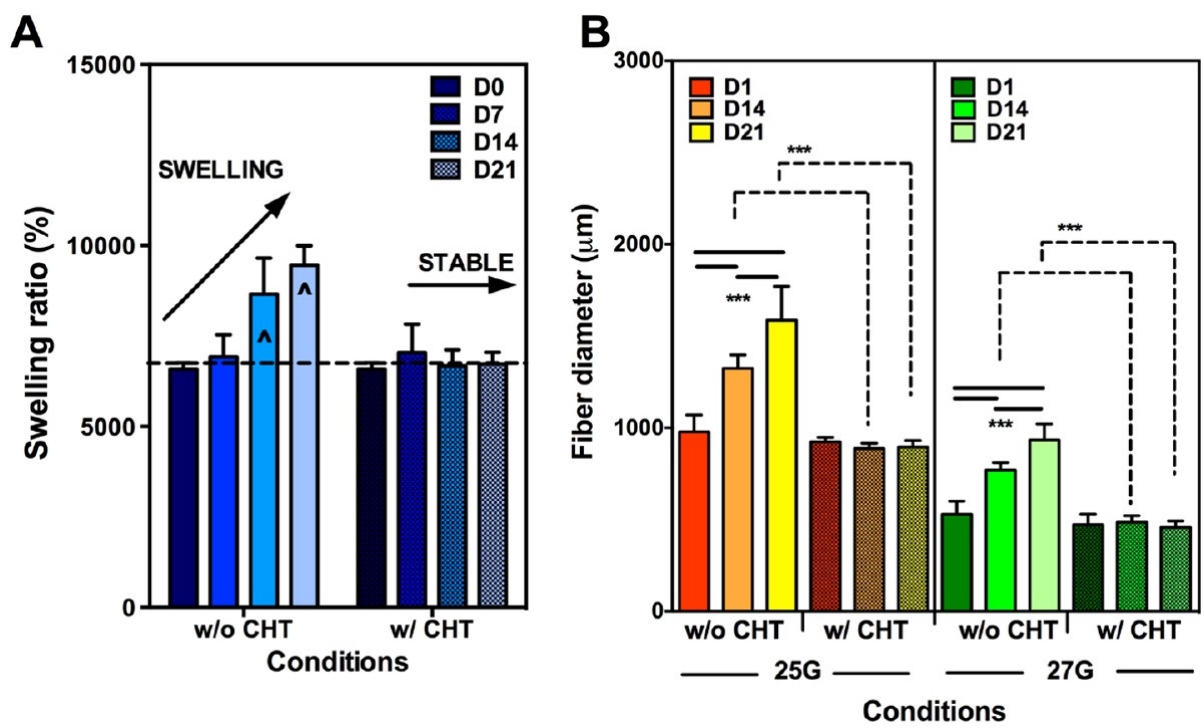

Figure 3. Swelling behavior of $\kappa$-CA fibers, without (w/o) and with (w/) CHT coating, in culture medium (DMEM). (A) Swelling ratio increases over time for the fibers without $\mathrm{CHT}$ coating. On the other side, the CHT coating stabilizes the hydrogel fibers, hampering their swelling. Values correspond to average $(n=3) \pm$ standard deviation. " $\wedge$ " corresponds to a statistical difference $(p<0.05)$ when compared to day 0 and day 7 values of the $\kappa$-CA fibers without coating. (B) The diameter of the developed fibers is a direct consequence of the swelling behavior. Thus, the uncoated $\kappa$-CA fibers show a significant increase in the fiber diameter along time $(* * * p<0.001)$, while the diameter of coated fibers remains constant.

324 Using needle gauges ranging from 18 to $27 \mathrm{G}$, it was possible to 325 obtain fibers with different diameters, directly proportional with 326 the internal diameter of the needles $(838-210 \mu \mathrm{m})$. The fibers 327 produced within these settings had a diameter ranging from 500 328 to $1250 \mu \mathrm{m}$ (Figure 1B), making them appealing for further 329 applications. ${ }^{13,14,43}$ These trends were in agreement with 330 previous reports for hydrogel fibers obtained via wet spinning ${ }^{18}$ 331 or other techniques. ${ }^{14}$

332 Taking into consideration that a microsized architecture 333 enables nutrients and oxygen diffusion, crucial for the cellular 334 performance, we decided to further explore the potential $\kappa$-CA 335 fibers with the smallest diameters, obtained by the extrusion of $\kappa$ $336 \mathrm{CA}$ solutions through the $25 \mathrm{G}$ and $27 \mathrm{G}$ needles.

337 3.2. $\kappa$-CA Fibers Can Be Coated with CHT through 338 Polyelectrolyte Complexation. Although encouraging results 339 have shown the potential of using $\kappa$-CA in TE applications, the 340 ionically cross-linked $\kappa$-CA hydrogels exhibit high swelling ratios. 341 It is possible that the culture medium destabilizes the physically 342 cross-linked network, as a result of the uncontrollable and 343 permanent exchange of $\mathrm{K}^{+}$ions with other positive ions present 344 in the physiological environment. ${ }^{24}$ This allows the inner 345 network to continuously expand, and consequently become 346 loose and mechanically weak. ${ }^{25,44}$ If one envisions the prolonged 347 culture periods or the maintenance of initial microfeatures of the 348 hydrogels, such as shape, size, and stability, the reinforcement of 349 the structure is an impetus.

350 Several methodologies have been employed, ${ }^{18,25,27}$ including 351 the formation of polyelectrolyte complexes formed by the ionic 352 interaction between oppositely charged polyelectrolytes. We 353 hypothesized that, by coating the $\kappa$-CA hydrogels fibers with $354 \mathrm{CHT}$, we would be able to stabilize the fibers structure. CHT, a $355 \beta$-1,4-linked D-glucosamine and $N$-acetyl-D-glucosamine, is one 356 of the few positively charged natural-origin polysaccharides and, 357 thus, is widely used as a polycation for the formation of 358 polyelectrolyte complexes. Additionally, its appealing properties 359 such as biocompatibility, biodegradability, low toxicity, and 360 relatively low production cost from abundant sources endorse its 361 use for TE strategies. ${ }^{45}$
Since the amino groups of CHT have a $\mathrm{p} K_{\mathrm{a}}$ value of $6.5-6.8,{ }^{46}{ }_{362}$ their protonation occurs in acidic solutions with a charge density 363 dependent on $\mathrm{pH}$ and degree of acetylation. Besides causing 364 CHT to become water-soluble, the protonated groups can 365 readily bind to the negatively charged groups (carboxylic acid, 366 hydroxyl, or sulfate) of other polymers, to form polyelectrolyte 367 complexes. Thus, our hypothesis was that the protonated amino 368 groups of CHT would bind to the available negatively charged 369 sulfate groups of $\kappa$-CA and form an acid-base type of 370 polyelectrolyte complex. Therefore, we have deliberately chosen 371 shorter gelation times for the formation of $\kappa$-CA fibers ( $10 \mathrm{~min} 372$ over standard $30 \mathrm{~min}^{18,41}$ ), so that the fiber shape was retained, 373 without neutralizing all sulfate groups.

374

However, since the growth of cells is usually optimal when the 375 microenvironment is buffered at a $\mathrm{pH}$ in the range $7.2-7.4,{ }^{47}$ the 376 low $\mathrm{pH}$ values at which $\mathrm{CHT}$ dissolves might compromise their 377 viability. Thus, we explored the possibility of increasing the $\mathrm{pH}$ of 378 $\mathrm{CHT}$ solutions without jeopardizing their solubility, by adding a 379 week base, $\beta \mathrm{GP},{ }^{36}$ to the solutions. We noticed that, by gradually 380 adding $\beta \mathrm{GP}$ to the $\mathrm{CHT}$ solutions, the $\mathrm{pH}$ slowly increased 381 without evident signs of precipitation (Figure 2A). Nevertheless, $382 \mathrm{f} 2$ upon reaching the $\mathrm{p} K_{\mathrm{a}}$ value, precipitation occurred.

383

One concern associated with the increase of $\mathrm{pH}$ was that the 384 amino groups of $\mathrm{CHT}$ would be deionized, which would weaken 385 their binding affinity to the oppositely charged $\kappa$-CA, jeopard- 386 izing the stability of the layer. To determine whether this was the 387 case, we evaluated the electrical charge of the CHT solution upon 388 addition of $\beta \mathrm{GP}$, and consecutively upon increase of $\mathrm{pH}$, by 389 measuring the zeta potential. As expected, with the increase of 390 the $\mathrm{pH}$, the overall charge of the CHT solution decreased, 391 though still remaining within the positive range (approximately 392 $+10 \mathrm{eV}$ ) (Figure 2B). These findings indicate that with the $\mathrm{pH} 393$ modification, the protonation of amino groups still occurs, and 394 consequently $\mathrm{CHT}$ would continue to act as a polycation, being 395 suitable for PEC with $\kappa$-CA. In the perspective of coating cell- 396 loaded $\kappa$-CA fibers, pHof 5.5, where no signs of precipitation 397 were observed and the overall charge of CHT was positive, was 398 considered the most suitable condition. 


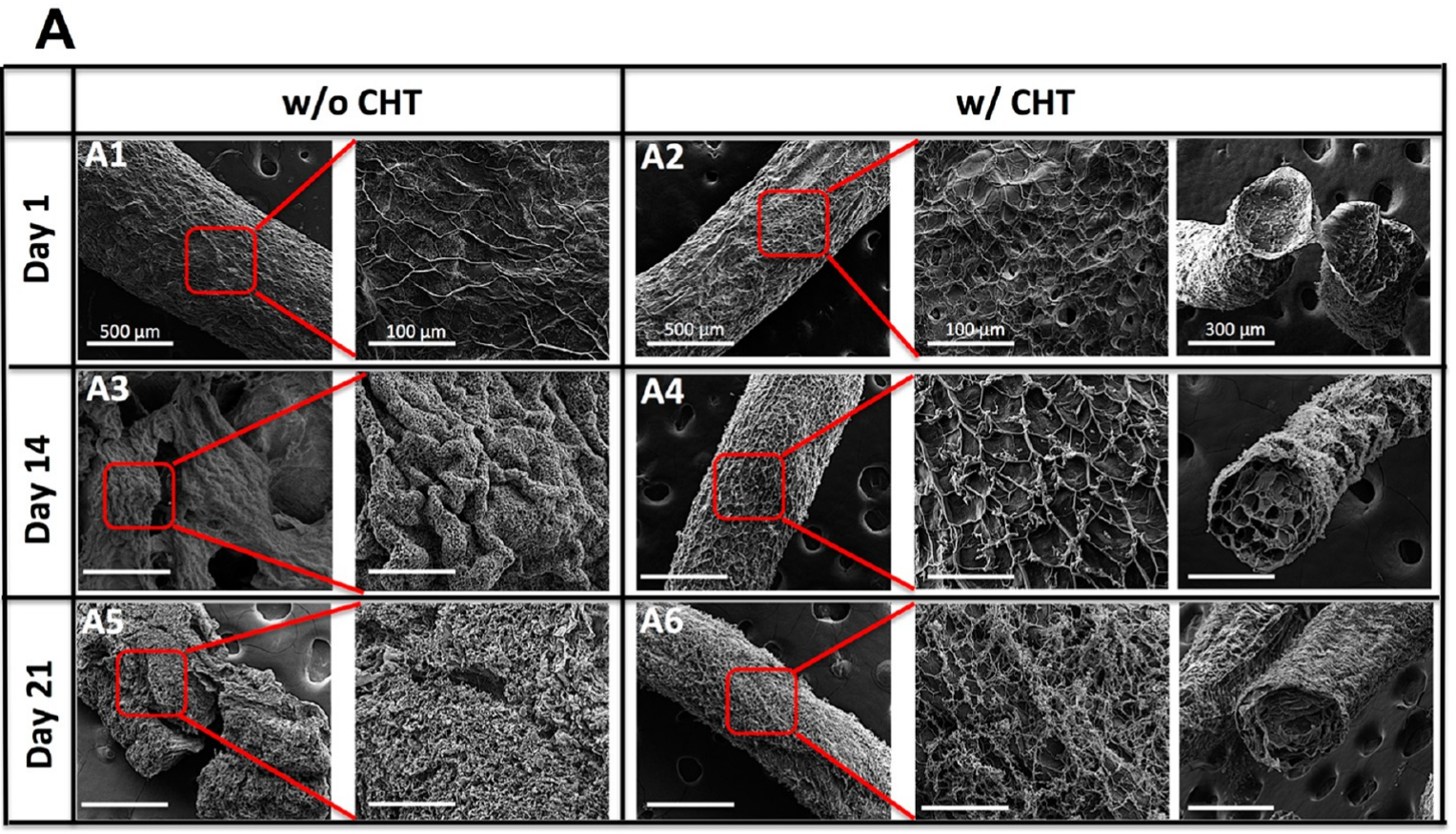

\begin{tabular}{|c|c|c|c|c|c|c|c|}
\hline \multirow{2}{*}{$\begin{array}{c}\text { Time } \\
\text { (days) }\end{array}$} & \multicolumn{7}{|c|}{ EDX/EDS elemental analysis (\%wt) } \\
\hline & Condition & C & 0 & $\mathbf{S}$ & $\mathbf{K}$ & $\mathbf{C l}$ & $\mathbf{N}$ \\
\hline \multirow{2}{*}{1} & w/o CHT & 26.01 & 25.82 & 4.49 & 9.64 & 22.32 & - \\
\hline & w/ CHT & 35.40 & 31.67 & 4.04 & 1.33 & 13.19 & 5.79 \\
\hline \multirow{2}{*}{14} & w/o CHT & 31.27 & 18.8 & 3.85 & 7.90 & 25.15 & - \\
\hline & w/ CHT & 41.62 & 22.38 & 5.15 & 1.81 & 12.04 & 7.01 \\
\hline \multirow{2}{*}{21} & w/o CHT & 41.54 & 13.5 & 2.58 & 1.71 & 26.67 & - \\
\hline & w/ CHT & 24.79 & 12.88 & 3.64 & 1.28 & 35.25 & 4.47 \\
\hline
\end{tabular}

Abbreviations: $w / 0=$ without; $w /=$ with.

Figure 4. Physicochemical characterization of the freeze-dried fibers. (A) SEM micrographs depict the alteration of fiber morphology in culture medium (DMEM) over time. The structure of the uncoated fibers is not stable and disintegrates after 21 days (A1-A3-A5), while the CHT coating exhibits a protective role that hinders the disintegration of the fibers and therefore enhances their stability (A2-A4-A6). (B) The presence of CHT was evaluated along time by tracing the amounts of nitrogen (from the $-\mathrm{NH}_{2}$ groups of $\mathrm{CHT}$ ) present on the surface of the fibers. The element was detected until day 21, suggesting that CHT was still present on the fibers. The defined squares in the SEM micrographs represent the area where the magnification and elemental analysis were performed.

400 The formation of the polyelectrolyte complexes relies on the 401 interaction between the available negatively charged sulfate 402 groups of $\kappa$-CA and the protonated amino groups of $\mathrm{CHT}$ 403 (Figure 2C). Thus, the immersion of $\kappa$-CA fibers in the 404 optimized CHT solution led to the formation of a localized CHT 405 nanosized layer, which was in agreement with previously 406 published data. ${ }^{29-33}$ The presence of the CHT coating was 407 confirmed by the intense pink coloration observed only on the 408 coated fibers after staining with Eosin Y, which specifically stains $409 \mathrm{CHT}^{48}$ (Figure 2C-C1 inset). From a practical standpoint, $410 \mathrm{CHT}$ coated fibers, when compared with the uncoated fibers, 411 were easier to separate and handle despite their small diameters. $412 \quad 3.3$. CHT Coated $\kappa$-CA Fibers Depict Improved Stability. 413 In the context of TE, it is important to evaluate the behavior of 414 these fibers in conditions similar to the in vivo microenviron415 ment. Swelling properties of hydrogels directly affect the overall 416 features of the polymeric network (shape, size, and mechanical stability). Thus, we aimed to evaluate to which extent the 417 swelling ratio of the developed fibers affects their overall 418 diameter, and whether the presence of CHT provides stability 419 to the fibers. We performed swelling studies by maintaining the 420 coated and uncoated fibers in culture medium, which consists of 421 a collection of ions and proteins that could destabilize the $\kappa$-CA ${ }_{422}$ hydrogel network. ${ }^{41}$

Our findings showed that the swelling ratios of ionically cross- 424 linked $\kappa$-CA fibers significantly increased with time $(p<0.001){ }_{425}$ (Figure 3A). The destabilization of the ionically cross-linked $\kappa-426 \mathrm{f3}$ CA network is likely to occur due to the continuous exchange of 427 $\mathrm{K}^{+}$ions entrapped within the network with other ions present in 428 the culture medium. In fact, similar results have been reported for 429 ionically cross-linked alginate ${ }^{24}$ and gellan gum, ${ }^{44}$ where 430 prolonged immersion in culture medium led to the weakening 431 of the network and, consequently, to its collapse. 

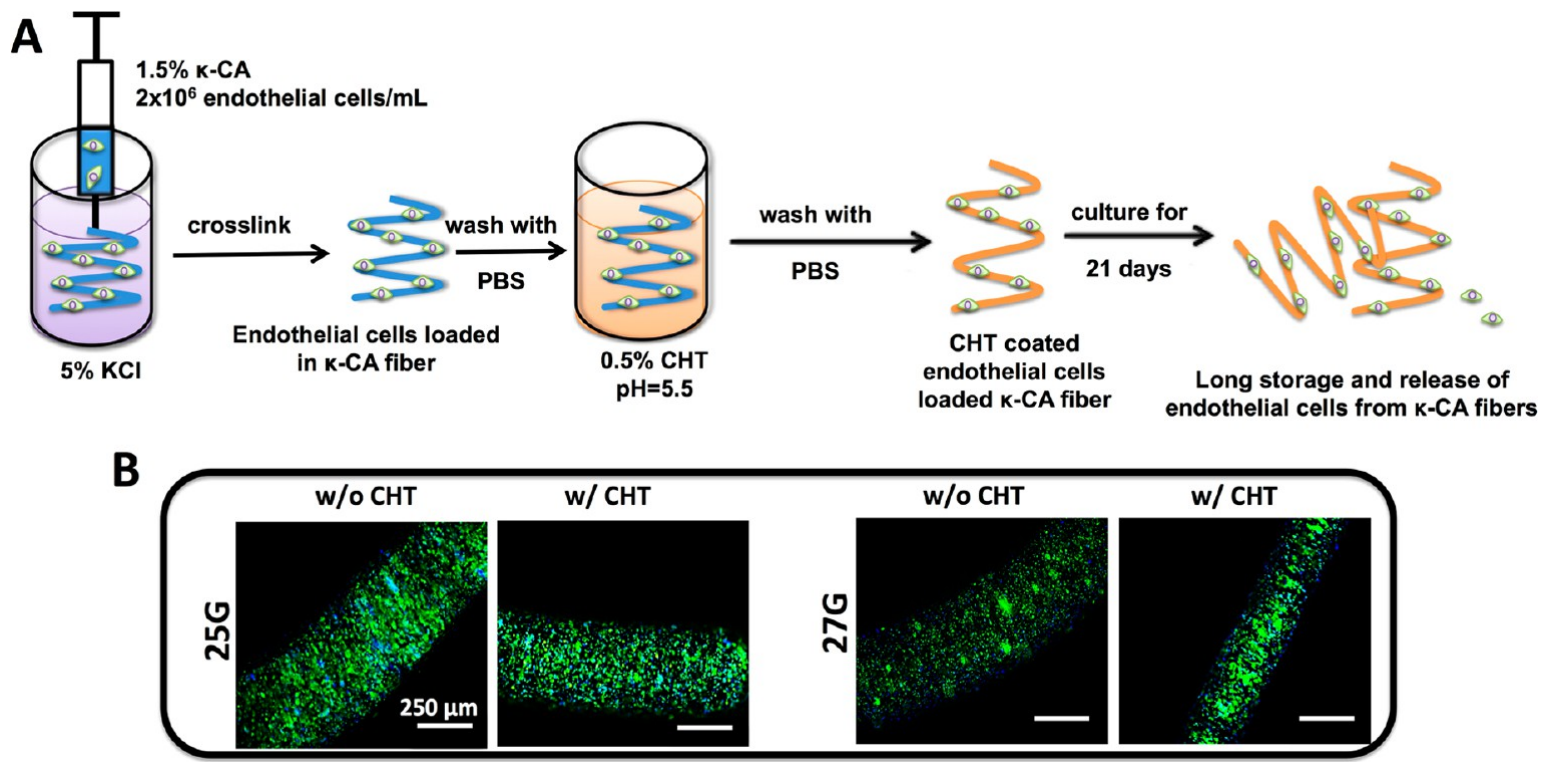

Figure 5. Encapsulation of SSEA- $4^{+}$hASCs-derived ECs into $\kappa$-CA fibers. (A) Schematics of the encapsulation procedure and experimental setup. (B) The assessment of cell viability (live (green)/dead (red)) $24 \mathrm{~h}$ after encapsulation shows that the cells were not affected by the processing method, nor by the size and CHT coating of the fibers. Cell nuclei were counterstained with DAPI (blue).

433 However, CHT coated fibers exhibited a stable swelling ratio 434 over time, significantly inferior to that of the uncoated fibers $(p<$ 435 0.001). This behavior can be due to the strong protective coating 436 layer, preventing the fiber swelling. A similar behavior was 437 described in other studies, concerning the use of CHT in 438 combination with alginate to form a layer of wrapping alginate 439 beads for the controlled release of drugs. ${ }^{29}$

440 As a consequence, the diameter of CHT coated fibers was 441 maintained constant over time, while the uncoated fibers 442 exhibited an increase in their diameter (Figure 3B). Even 443 more, in terms of manipulation, the uncoated fibers became soft 444 and easy to break, while the coated ones were stable over the long 445 term and easy to handle, without obvious damage to their 446 integrity.

447 The closer evaluation of the fiber morphology by SEM 448 revealed that the uncoated fibers started to lose their integrity 449 around day 14 (Figure 4A1-3-5), as predicted by the swelling 450 ratio results (Figure 3A). By contrast, the CHT coated fibers 451 exhibited a rougher surface, displaying a mesh-like wrapping 452 around an open-pore $\kappa$-CA core. These fibers maintained their 453 shape, size, and integrity until day 21 , when small cracks become 454 visible on their surface (Figure 4A2-4-6). The presence of the $455 \mathrm{CHT}$ coating was further demonstrated by tracing the $\mathrm{N}$ content 456 on the surface of the CHT-coated $\kappa$-CA fibers by EDS/EDX 457 (Figures $4 \mathrm{~B}$ ) over time. Its detection up to 21 days suggested that 458 the $\mathrm{CHT}-\kappa$-CA electrostatic interactions are strong enough to 459 withstand the presence of other ions and proteins present in the 460 culture medium, leading to a robust and stable structure.

$461 \quad 3.4$. CHT Coating of the Cell-Loaded $\kappa$-CA Fibers Does 462 Not Affect the Endothelial Differentiated SSEA-4 ${ }^{+}$hASCs 463 Phenotype in the Long Term. Recently, several cell types, 464 such as human adipose derived stem cells, human nasal 465 chondrocytes, ATDC5 chondrocytic cell line, and L929 466 fibroblast cell line, were encapsulated within k-CA hydrogels, 467 processed in different shapes (fibers, beads, discs), with positive 468 results in terms of viability, cellular metabolism, proliferation, 469 and differentiation. ${ }^{41,49,50}$ Herein, for the first time, we proposed 470 the encapsulation of microvascular-like ECs within k-CA hydrogel fibers as building blocks of vascularized bone tissue 471 engineered constructs. Pre-vascularization of engineered con- 472 structs by using progenitor/endothelial cells has been considered 473 the most promising strategy to address the vascularization of 474 large constructs. ${ }^{51}$ However, the use of human cells has been 475 hampered by the limited sources and reduced yields. ${ }^{52}$ Our 476 earlier work $^{34}$ has shown that the SSEA- $4^{+}$subpopulation, 477 selected among the heterogeneous stromal vascular fraction of 478 human adipose tissue, can be differentiated toward both 479 endothelial and osteogenic lineages. Moreover, SSEA- 480 $4^{+}$hASCs-derived ECs hold features of microvascular ECs that 481 trigger the formation of 3D vascular networks within engineered 482 constructs. Therefore, we have taken advantage of our 483 knowledge to obtain ECs from SSEA-4 ${ }^{+}$subpopulation 484 (Supporting Information (SI) Figure 1) to develop cell-laden 485 $\kappa$-CA-based hydrogel fibers to be used as $3 \mathrm{D}$ cellular carriers. 486 SSEA- $4^{+} h A S C s-d e r i v e d ~ E C s$ were encapsulated within the $\kappa$-CA 487 and $\mathrm{CHT}$ coated $\kappa$-CA fibers with 2 different diameters and 488 cultured for a period of 21 days (Figure 5A).

As the encapsulation procedure requires several steps that 490 could lead to reduced cellular performance, ${ }^{53,54}$ the direct effect 491 of the experimental conditions over the viability of the 492 encapsulated cells was assessed. Independently of the size and 493 whether fibers were coated or not, the majority of the 494 encapsulated cells were viable (average $>65 \%$, SI Figure 2) and 495 homogeneously distributed within the fibers (Figure 5B), 496 suggesting that the production of fibers (ionotropic gelation 497 and PEC) was mild enough.

Additionally, along the culture time and independently of the 499 conditions, viable cells were predominantly observed within the 500 fibers (Figure 6). A slight decrease in cellular viability was $501 \mathrm{fo}$ noticed, so that by day 21 , viable cells comprised $60 \%$ of the total 502 cells (SI Figure 2). However, this drop was not as significant as 503 reported in previous studies that associated this outcome with 504 the slow diffusion of nutrients caused by the sample thickness 505 and/or cross-linking mechanism..$^{25,55,56}$ It might be due to the 506 fact that fiber-shaped hydrogels were characterized by a larger 507 surface area than disc shaped units, and as a consequence, a faster 508 


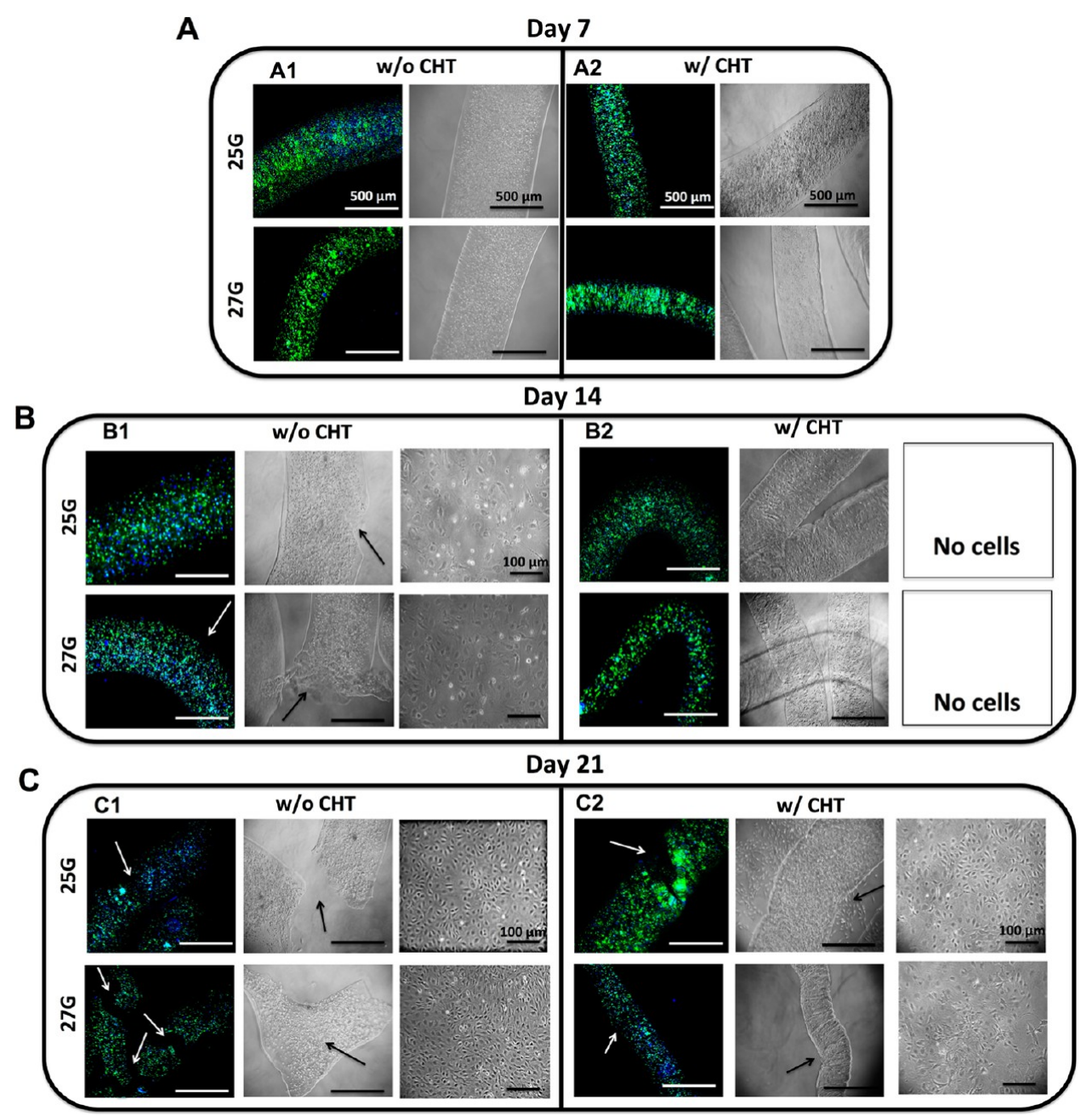

Figure 6. Evaluation of the cell-laden $\kappa$-CA hydrogel fibers along 21 days of culture. The viability of encapsulated cells (live/dead/) was maintained during the culture time in all conditions. (A) At day 7, independently of the conditions, fibers were intact, without displaying signs of disintegration. (B1) At day 14, cells escaped the uncoated fibers and small endothelial-like colonies could be observed at the bottom of the well. By day 21 (C1), fibers lose their integrity (arrows), allowing further release of cells into the well plate. Contrarily, the CHT coating (w/CHT) delayed the disintegration of the fibers, hence the release of the cells (B2 and C2). At day 21, the coated fiber diameter did not alter; however, small cracks (arrows) were observed along the fibers allowing the release of the cells. Cell nuclei were counterstained with DAPI (blue).

509 diffusion of nutrients and oxygen to the core of the sample could 510 occur. ${ }^{57}$ It has also been suggested that the ions needed for 511 hydrogel formation may influence the cellular viability; never512 theless, the fibers reported herein are formed via a partial cross513 linking process, by shorter exposure times to $\mathrm{K}^{+}$treatment than 514 previously reported. ${ }^{18,58}$ The additional CHT coating, that was 515 shown to prevent the fibers from swelling, did not affect viability. 516 From the SEM images it was possible to notice that the CHT 517 layer was displayed as a mesh wrapped around the fiber, 518 preventing it from swelling, but enabling diffusion.

519 The optical analysis of the cell-loaded uncoated fibers showed 520 an increase in their diameter and subsequent disintegration, in 521 agreement with the results obtained with acellular fibers. 522 Concisely, at day 7, uncoated fibers were intact, with a well523 delimited smooth surface; however, at day 14, they presented 524 signs of disintegration. This allowed the entrapped cells to 525 "escape" from the fibers and adhere to the well and proliferate 526 (Figure 6A1-C1). In opposition, the CHT coating delayed the 527 disintegration of fibers, as they maintained their initial diameter 528 and their surface did not present disruptions up to day 14 (Figure 529 6A2-B2). Only at the last time point did small cracks become visible, and consequently, EC colonies were observed on the 530 bottom of the well (Figure 6C2).

Beside potentially affecting cellular viability, ${ }^{7}$ cell encapsula- 532 tion procedures and prolonged culture might also compromise 533 the cell's phenotype or functionality. ${ }^{20,59}$ In order to address this 534 question, cells were retrieved from the fibers, after culturing for 535 21 days, and seeded into tissue culture flasks. Cells were able to 536 adhere and organize into small endothelial-like colonies (Figure $537 \mathrm{f7}$ 7A) and, more importantly, maintained their ability to form $538 \mathrm{f7}$ tubular-like structures when seeded on Matrigel (Figure 7B). 539 Moreover, when screened for the initial cell surface marker panel, 540 cells were shown to be $\mathrm{CD} 105^{+} / \mathrm{CD}_{3}{ }^{+} / \mathrm{CD} 31^{+} / \mathrm{CD} 34^{+} / 541$ $\mathrm{CD} 45^{-} / \mathrm{CD}^{-} 0^{-}$, a signature that matches the one registered 542 prior encapsulation. Taken together, these results show that the 543 encapsulation process, followed by the prolonged culture of cells 544 within fibers and their retrieval, did not affect the phenotype and 545 in vitro functionality of ECs.

Overall, as $\kappa$-CA fibers exhibit high swelling ratios, the 547 weakening of the network occurred and, consequently, cells were 548 able to escape from the fibers. CHT coating maintained the fiber 549 diameter, delaying their disintegration and the consequent 550 release of cells into the surrounding environment. Nonetheless, 551 

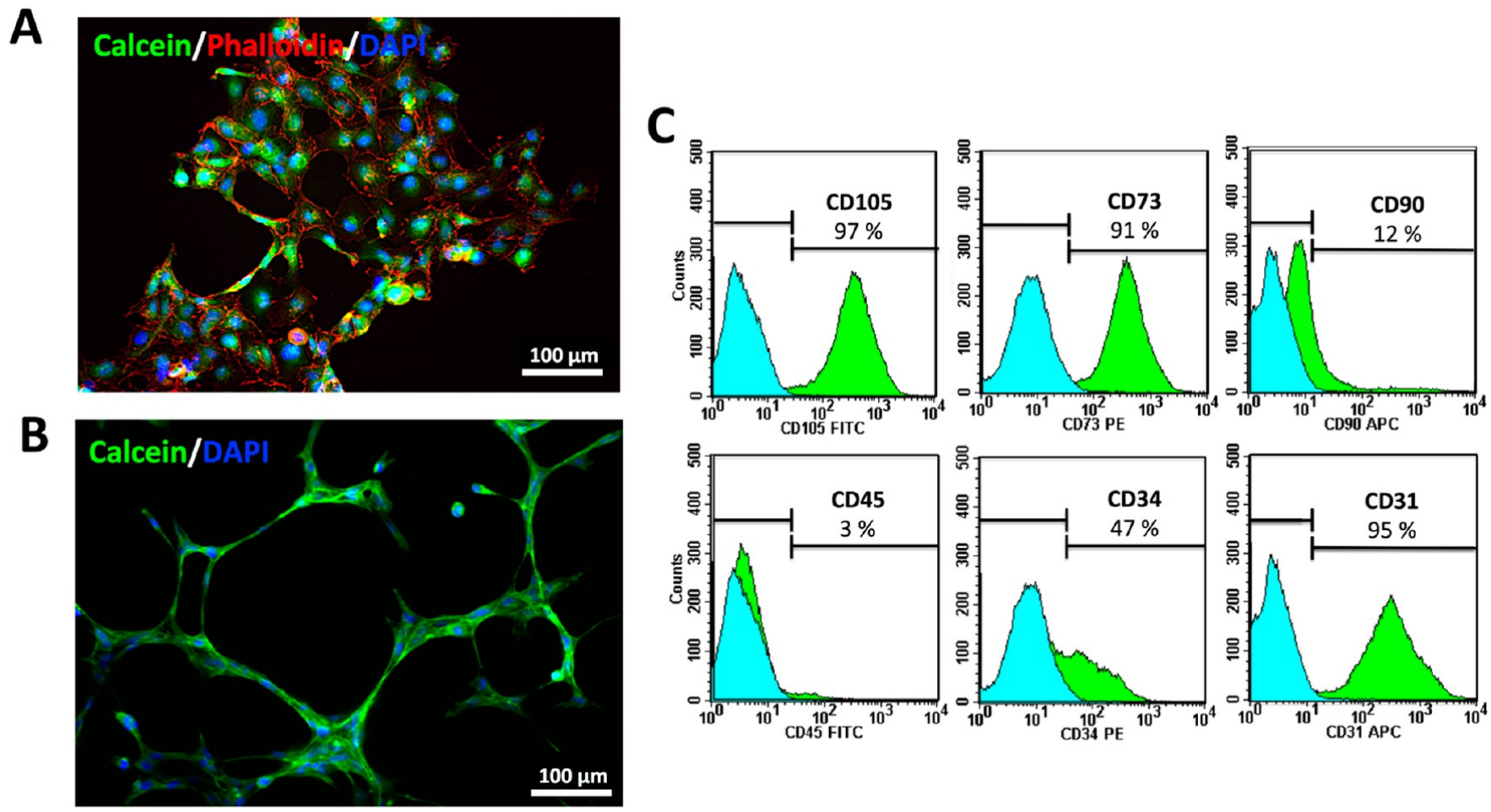

Figure 7. Endothelial phenotype of cells retrieved from the fibers after 21 days of culture. (A) After retrieval of cells through degradation of the fibers, and their subsequent seeding, cells adhered and formed small colonies, characterized by close contact between cells (live/cytoskeleton/nuclei). (B) Cells maintained their ability to form tubular-like structures (green) when seeded on Matrigel (C) and the expression of the initial endothelial phenotype-associated panel of markers. Cell nuclei were counterstained with DAPI (blue).

552 the CHT layer was still permissive to culture medium, allowing 553 the diffusion of its components, as suggested by the subsequent 554 remarkable results concerning the viability and phenotype of 555 encapsulated cells. These findings further demonstrate the 556 potential of using these fibers as EC carriers within different TE 557 constructs.

$558 \quad$ 3.5. Cell-Loaded $\kappa$-CA-Based Fibers Can Act as Building 559 Blocks within 3D Hydrogel Constructs. In vitro 3D co560 culture platforms provide a scalable and flexible approach to 561 evaluate the indirect interactions between multiple-cell types in 562 highly defined settings, enabling us to address specific cellular 563 behaviors and the formation of complex tissue-like analogs. 564 Simultaneously, the integration and assembly of cell-laden 565 templates as building blocks within $3 \mathrm{D}$ constructs may allow 566 the development of replicas of complex tissues to use in TE 567 approaches. Herein, we proposed a straightforward method to 568 integrate the $\kappa$-CA-based fibers enclosing SSEA- $4^{+}$hASCs569 derived ECs, within a 3D hydrogel containing SSEA- $4^{+}$hASCs570 derived osteoblast-like cells, as heterotypic constructs of interest 571 for bone TE. Within this approach, we took advantage of the ease 572 preparation of cell-loaded $\kappa$-CA fibers, to recreate the cellular 573 indirect communication at a $3 \mathrm{D}$ scale. Thus, cell-loaded fibers, 574 used as building blocks, were assembled within a $\kappa$-CA hydrogel 575 disc by simply laying them in a Petri dish (Figure 8 ). They could 576 be singled out or stacked in a random or organized manner 577 (Figure 8A). When the cell-laden fibers were integrated in a 578 hydrogel disc containing osteoblast-like cells, the formation of a 579 3D heterotypic platform with a defined cellular topography 580 suitable for bone-vascularization strategies was achieved (Figure $5818 \mathrm{~B}-\mathrm{C}$ ). The proposed system was entirely formed by a $\kappa$-CA 582 matrix, with a controlled spatial distribution of the two cell types 583 derived from the same source. The fibers are expected to act both as cell-reservoirs and structurally defined arrangements in the 584 surrounding matrix. Within the context of bone TE, further 585 experiments aim to deeply assess the cellular interactions within 586 the $3 \mathrm{D}$ structure and the consequent outcome, both concerning 587 the outflow of ECs triggered by the crosstalk with osteoblast 588 cells, $^{60,61}$ as well as osteogenic matrix deposition and 589 mineralization. This could eventually lead to the organization 590 of ECs within the osteoblast-containing matrix, leading to an 591 improvement in the osteogenic outcome.

For the immediate integration of fibers in a secondary matrix, 593 the $\mathrm{CHT}$ coating does not provide additional advantages, as the 594 secondary matrix will already hold the fibers in place. 595 Nevertheless, the CHT coating proves to be crucial for 596 prolonged culture periods that are relevant when using this 597 approach to study indirect interactions between multiple-cell 598 types and/or for those 3D systems in which the accuracy of the 599 initial parameters (shape, size, swelling, mechanical stability) is 600 crucial. For instance, it could be possible to create a $3 \mathrm{D}$ co-culture 601 system based on coated cell-laden $\kappa$-CA fibers by seeding another 602 cell type on top of the fibers.

\section{CONCLUSIONS}

The development of chitosan $(\mathrm{CHT})$ reinforced $\kappa$-carrageenan 604 $(\kappa$-CA) fibers using a two-step procedure, under cell-friendly 605 experimental settings, aiming at homogeneous immobilization of 606 cells is herein reported. The diameter of the fibers could be easily 607 tuned by selecting the appropriate needle gauge during 608 processing. The presence of the CHT coating enhanced the 609 stability of the fibers and their diameter was maintained constant 610 by restraining their swelling. Moreover, these fibers support the 611 viability and functionality of encapsulated cells during long-term 612 culturing, enabling their use as cell-delivery systems within 3D 613 


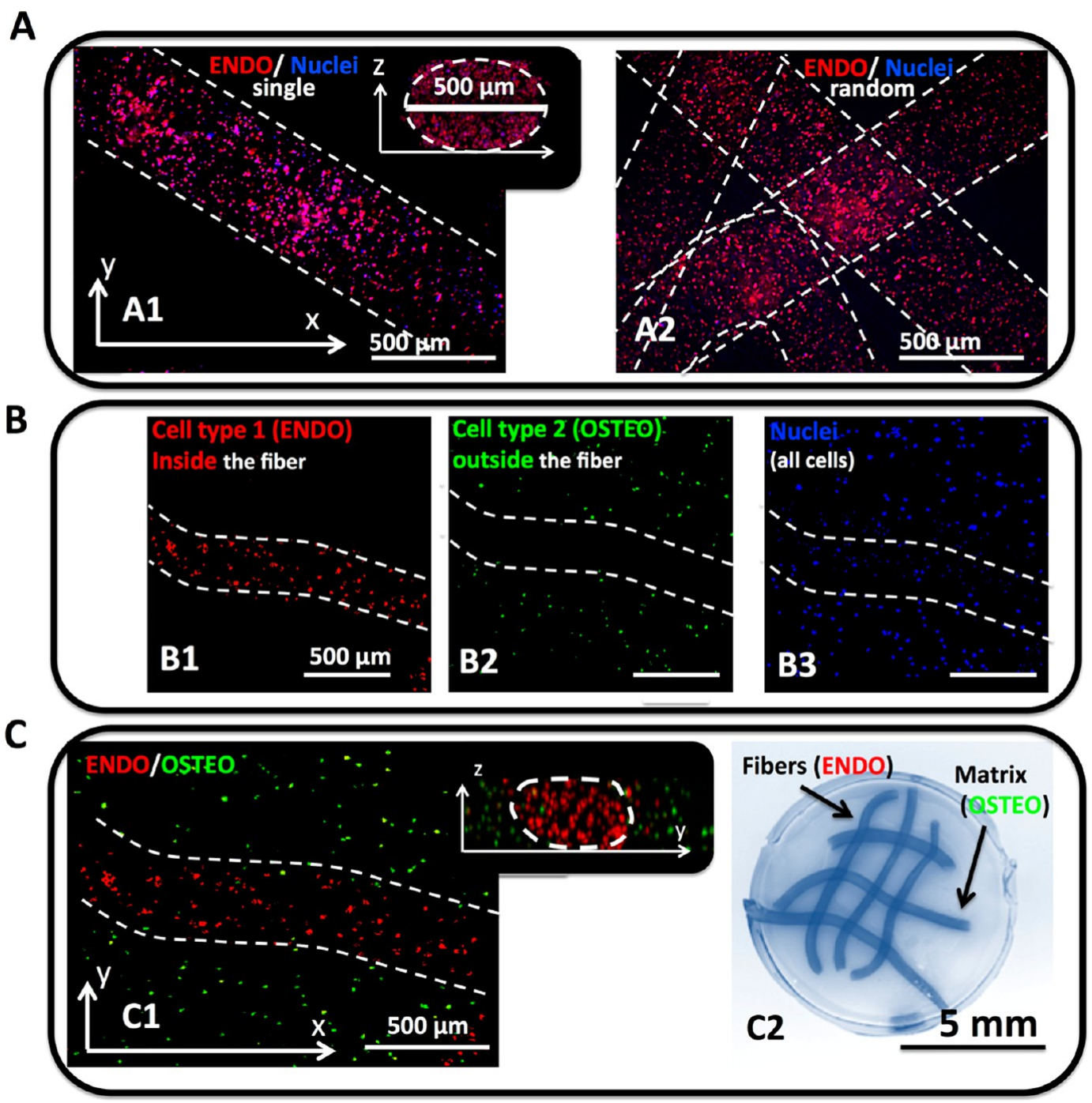

Figure 8. Proof of concept of the use of cell-loaded $\kappa$-CA-based fibers as building blocks within 3D $\kappa$-CA hydrogel constructs. Confocal laser scanning micrographs of (A1-2) encapsulated SSEA-4+hASCs-derived ECs (ENDO) within $\kappa$-CA fibers. Fibers can be (A1) singled out or (A2) randomly stacked. (B,C) Heterotypic 3D $\kappa$-CA-based structure. (B) The encapsulation of ENDO preceded the integration of the fibers within the $\kappa$-CA containing the SSEA-4+hASCs-derived osteoblast-like cells (OSTEO) localized outside the fiber. All cells (blue) were evenly distributed within the hydrogel. (C) 3D buildup of fiber stacks within a hydrogel disc consisted of a controlled spatial localization of two cell types within a single hydrogel matrix. (C1) The colocalization of ENDO and OSTEO is relevant for developing spatial controlled heterotypic systems aimed at vascularized bone TE approaches. Cell nuclei were counterstained with DAPI (blue). (C2) A macroscopic view of the $3 \mathrm{D}$ construct. The discs were manually manipulated without damage. $\kappa$ CA fibers were stained with methylene blue (dark blue).

614 tissue engineering constructs. Furthermore, using a bottom-up 615 approach, these fibers can be used as building blocks for the 616 development of suitable $3 \mathrm{D}$ platforms of independently 617 organized heterotypic cell-containing hydrogels, relevant for $618 \mathrm{TE}$ approaches.

\section{ASSOCIATED CONTENT}

\section{S Supporting Information}

621 Supplemental figures depict the endothelial phenotype of SSEA$6224^{+}$hASCs-derived ECs phenotype prior encapsulation and the 623 propidium iodide/DAPI images of fibers along time as well as the 624 quantification of the cellular viability. This material is available 625 free of charge via the Internet at http://pubs.acs.org.

\section{AUTHOR INFORMATION}

\section{Corresponding Author}

628 *E-mail: megomes@dep.uminho.pt.

\section{Notes}

The authors declare no competing financial interest.

\section{ACKNOWLEDGMENTS}

Authors thank the Portuguese Foundation for Science and 632 Technology (FCT) for the personal grants SFRH/BD/42968/ 633 2008 through the MIT-Portugal Program (SMM) and SFRH/ 634 $\mathrm{BD} / 64070 / 2009$ (EGP). The research leading to these results 635 has received funding from the European Union's Seventh 636 Framework Programme (FP7/2007-2013) under grant agree- 637 ment no REGPOT-CT2012-316331-POLARIS and MIT/ECE/ 638 $0047 / 2009$ project.

\section{REFERENCES}

(1) Berthiaume, F.; Maguire, T. J.; Yarmush, M. L. Tissue engineering 641 and regenerative medicine: history, progress, and challenges. Annu. Rev. 642 Chem. Biomol. Eng. 2011, 2, 403-30. 
644 (2) Kannan, R. Y.; Salacinski, H. J.; Sales, K.; Butler, P.; Seifalian, A. M. 645 The roles of tissue engineering and vascularisation in the development 646 of micro-vascular networks: a review. Biomaterials 2005, 26 (14), 185764775 .

648 (3) Bae, H.; Puranik, A. S.; Gauvin, R.; Edalat, F.; Carrillo-Conde, B.; 649 Peppas, N. A.; Khademhosseini, A. Building vascular networks. Sci. 650 Transl. Med. 2012, 4 (160), 160ps23.

651 (4) Lovett, M.; Lee, K.; Edwards, A.; Kaplan, D. L. Vascularization 652 strategies for tissue engineering. Tissue Eng., Part B 2009, 15 (3), 35365370.

654 (5) Phelps, E. A.; Garcia, A. J. Engineering more than a cell: 655 vascularization strategies in tissue engineering. Curr. Opin. Biotechnol. 656 2010, 21 (5), 704-9.

657 (6) Geckil, H.; Xu, F.; Zhang, X.; Moon, S.; Demirci, U. Engineering 658 hydrogels as extracellular matrix mimics. Nanomedicine 2010, 5 (3), 659 469-84.

660 (7) Drury, J. L.; Mooney, D. J. Hydrogels for tissue engineering: 661 scaffold design variables and applications. Biomaterials 2003, 24 (24), $662 \quad 4337-51$.

663 (8) Malafaya, P. B.; Silva, G. A.; Reis, R. L. Natural-origin polymers as 664 carriers and scaffolds for biomolecules and cell delivery in tissue 665 engineering applications. Adv. Drug Delivery Rev. 2007, 59 (4-5), 20766633.

667 (9) Fedorovich, N. E.; Wijnberg, H. M.; Dhert, W. J.; Alblas, J. Distinct 668 tissue formation by heterogeneous printing of osteo- and endothelial 669 progenitor cells. Tissue Eng., Part A 2011, 17 (15-16), 2113-21.

670 (10) Kang, E.; Jeong, G. S.; Choi, Y. Y.; Lee, K. H.; Khademhosseini, A.; 671 Lee, S. H. Digitally tunable physicochemical coding of material 672 composition and topography in continuous microfibres. Nat. Mater. 6732011,10 (11), 877-83.

674 (11) Aubin, H.; Nichol, J. W.; Hutson, C. B.; Bae, H.; Sieminski, A. L.; 675 Cropek, D. M.; Akhyari, P.; Khademhosseini, A. Directed 3D cell 676 alignment and elongation in microengineered hydrogels. Biomaterials 677 2010, 31 (27), 6941-6951.

678 (12) Liu, Y.; Sakai, S.; Taya, M. Production of endothelial cell679 enclosing alginate-based hydrogel fibers with a cell adhesive surface 680 through simultaneous cross-linking by horseradish peroxidase-catalyzed 681 reaction in a hydrodynamic spinning process. J. Biosci. Bioeng. 2012, 114 682 (3), 353-9.

683 (13) Takei, T.; Sakai, S.; Ijima, H.; Kawakami, K. Development of 684 mammalian cell-enclosing calcium-alginate hydrogel fibers in a co685 flowing stream. Biotechnol. J. 2006, 1 (9), 1014-7.

686 (14) Sakai, S.; Yamaguchi, S.; Takei, T.; Kawakami, K. Oxidized 687 alginate-cross-linked alginate/gelatin hydrogel fibers for fabricating 688 tubular constructs with layered smooth muscle cells and endothelial cells 689 in collagen gels. Biomacromolecules 2008, 9 (7), 2036-41.

690 (15) Enea, D.; Henson, F.; Kew, S.; Wardale, J.; Getgood, A.; Brooks, 691 R.; Rushton, N. Extruded collagen fibres for tissue engineering 692 applications: effect of crosslinking method on mechanical and biological 693 properties. J. Mater. Sci. Mater. Med. 2011, 22 (6), 1569-78.

694 (16) Oliveira, J. T.; Martins, L.; Picciochi, R.; Malafaya, P. B.; Sousa, R. 695 A.; Neves, N. M.; Mano, J. F.; Reis, R. L. Gellan gum: a new biomaterial 696 for cartilage tissue engineering applications. J. Biomed. Mater. Res., Part A 697 2010, 93 (3), 852-63.

698 (17) Berger, J.; Reist, M.; Mayer, J. M.; Felt, O.; Peppas, N. A.; Gurny, 699 R. Structure and interactions in covalently and ionically crosslinked 700 chitosan hydrogels for biomedical applications. Eur. J. Pharm. Biopharm. 701 2004, 57 (1), 19-34.

702 (18) Popa, E. G.; Gomes, M. E.; Reis, R. L. Cell delivery systems using 703 alginate-carrageenan hydrogel beads and fibers for regenerative 704 medicine applications. Biomacromolecules 2011, 12 (11), 3952-61.

705 (19) Kirsch, P. P. Carrageenan: a safe additive. Environ. Health Perspect. 7062002,110 (6), A288 author reply A288.

707 (20) Popa, E. G.; Reis, R. L.; Gomes, M. E. Chondrogenic phenotype 708 of different cells encapsulated in kappa-carrageenan hydrogels for 709 cartilage regeneration strategies. Biotechnol. Appl. Biochem. 2012, 59 (2), $710132-41$.

711 (21) Popa, E. G.; Rodrigues, M. T.; Coutinho, D. F.; Oliveira, M. B.; 712 Mano, J. F.; Reis, R. L.; Gomes, M. E. Cryopreservation of cell laden natural origin hydrogels for cartilage regeneration strategies. Soft Matter 713 2013, 9 (3), 875-885.

(22) Santo, V. E.; Frias, A. M.; Carida, M.; Cancedda, R.; Gomes, M. E.; 715 Mano, J. F.; Reis, R. L. Carrageenan-based hydrogels for the controlled 716 delivery of PDGF-BB in bone tissue engineering applications. 717 Biomacromolecules 2009, 10 (6), 1392-401.

(23) Salgueiro, A. M.; Daniel-da-Silva, A. L.; Fateixa, S.; Trindade, T. 719 kappa-Carrageenan hydrogel nanocomposites with release behavior 720 mediated by morphological distinct Au nanofillers. Carbohydr. Polym. 721 2013, 91 (1), 100-9.

(24) Kuo, C. K.; Ma, P. X. Maintaining dimensions and mechanical 723 properties of ionically crosslinked alginate hydrogel scaffolds in vitro. J. 724 Biomed. Mater. Res., Part A 2008, 84 (4), 899-907.

725

(25) Mihaila, S. M.; Gaharwar, A. K.; Reis, R. L.; Marques, A. P.; 726 Gomes, M. E.; Khademhosseini, A. Photocrosslinkable kappa- 727 carrageenan hydrogels for tissue engineering applications. Adv. 728 Healthcare Mater. 2013, 2 (6), 895-907.

(26) Hezaveh, H.; Muhamad, I. I.; Noshadi, I.; Shu Fen, L.; Ngadi, N. 730 Swelling behaviour and controlled drug release from cross-linked kappa- 731 carrageenan/NaCMC hydrogel by diffusion mechanism. J. Micro- 732 encapsulation 2012, 29 (4), 368-79.

733

(27) Amici, E.; Clark, A. H.; Normand, V.; Johnson, N. B. 734 Interpenetrating network formation in agarose-kappa-carrageenan gel 735 composites. Biomacromolecules 2002, 3 (3), 466-74. 736

(28) Granero, A. J.; Razal, J. M.; Wallace, G. G.; Panhuis, M. i. h. 737 Conducting gel-fibres based on carrageenan, chitosan and carbon 738 nanotubes. J. Mater. Chem. 2010, 20, 7953-7956.

(29) Colinet, I.; Dulong, V.; Mocanu, G.; Picton, L.; Le Cerf, D. Effect 740 of chitosan coating on the swelling and controlled release of a poorly 741 water-soluble drug from an amphiphilic and $\mathrm{pH}$-sensitive hydrogel. Int. J. 742 Biol. Macromol. 2010, 47 (2), 120-5.

743

(30) Chang, H. H.; Wang, Y. L.; Chiang, Y. C.; Chen, Y. L.; Chuang, Y. 744 H.; Tsai, S. J.; Heish, K. H.; Lin, F. H.; Lin, C. P. A novel chitosan- 745 gammaPGA polyelectrolyte complex hydrogel promotes early new bone 746 formation in the alveolar socket following tooth extraction. PLoS One 747 2014, 9 (3), e92362.

(31) Grenha, A.; Gomes, M. E.; Rodrigues, M.; Santo, V. E.; Mano, J. 749 F.; Neves, N. M.; Reis, R. L. Development of new chitosan/carrageenan 750 nanoparticles for drug delivery applications. J. Biomed. Mater. Res., Part A 751 2010, 92 (4), 1265-72.

752

(32) Piyakulawat, P.; Praphairaksit, N.; Chantarasiri, N.; Muangsin, N. 753 Preparation and evaluation of chitosan/carrageenan beads for 754 controlled release of sodium diclofenac. AAPS PharmSciTech 2007, 8755 (4), E97.

756

(33) Pinheiro, A. C.; Bourbon, A. I.; Medeiros, B. G. d. S.; Silva, L. s. H. 757 M. d.; Silva, M. C. H. d.; Carneiro-da-Cunha, M. G.; Coimbra, M. A.; 758 Vicente, A. n. A. Interactions between k-carrageenan and chitosan in 759 nanolayered coatings-Structural and transport properties. Carbohydr. 760 Polym. 2012, 87, 1081-1090.

(34) Mihaila, S. M.; Frias, A. M.; Pirraco, R. P.; Rada, T.; Reis, R. L.; 762 Gomes, M. E.; Marques, A. P. Human adipose tissue-derived SSEA-4 763 subpopulation multi-differentiation potential towards the endothelial 764 and osteogenic lineages. Tissue Eng., Part A 2013, 19 (1-2), 235-46. 765

(35) Signini, R.; Filho, S. P. C. Characteristics and properties of 766 purified chitosan in the neutral, acetate and hydrochloride forms. Polim. 767 Ciênc. Tecnol. 2001, 11, 58-64.

768

(36) Cho, J.; Heuzey, M. C.; Begin, A.; Carreau, P. J. Physical gelation 769 of chitosan in the presence of beta-glycerophosphate: the effect of 770 temperature. Biomacromolecules 2005, 6 (6), 3267-75. 771

(37) Huh, D.; Hamilton, G. A.; Ingber, D. E. From 3D cell culture to 772 organs-on-chips. Trends Cell Biol. 2011, 21 (12), 745-54. 773

(38) Hahn, M. S.; Miller, J. S.; West, J. L. Three-dimensional 774 biochemical and biomechanical patterning of hydrogels for guiding cell 775 behavior. Adv. Mater. 2006, 18 (20), 2679-2684. 776

(39) Oliveira, J. T.; Gardel, L. S.; Rada, T.; Martins, L.; Gomes, M. E.; 777 Reis, R. L. Injectable gellan gum hydrogels with autologous cells for the 778 treatment of rabbit articular cartilage defects. J. Orthop. Res. 2010, 28 (9), 779 1193-9. 
781 (40) Kim, I. L.; Khetan, S.; Baker, B. M.; Chen, C. S.; Burdick, J. A. 782 Fibrous hyaluronic acid hydrogels that direct MSC chondrogenesis 783 through mechanical and adhesive cue. Biomaterials 2013, 34 (22), 784 5571-80.

785 (41) Popa, E. G.; Caridade, S. G.; Mano, J. F.; Reis, R. L.; Gomes, M. E. 786 Chondrogenic potential of injectable kappa-carrageenan hydrogel with 787 encapsulated adipose stem cells for cartilage tissue-engineering 788 applications. J. Tissue Eng. Regen. Med. 2013, DOI: 10.1002/term.1683. 789 (42) Pereira, R. C.; Scaranari, M.; Castagnola, P.; Grandizio, M.; 790 Azevedo, H. S.; Reis, R. L.; Cancedda, R.; Gentili, C. Novel injectable gel 791 (system) as a vehicle for human articular chondrocytes in cartilage tissue 792 regeneration. J. Tissue Eng. Regen. Med. 2009, 3 (2), 97-106.

793 (43) Fedorovich, N. E.; De Wijn, J. R.; Verbout, A. J.; Alblas, J.; Dhert, $794 \mathrm{~W}$. J. Three-dimensional fiber deposition of cell-laden, viable, patterned 795 constructs for bone tissue printing. Tissue Eng., Part A 2008, 14 (1), 796 127-33.

797 (44) Coutinho, D. F.; Sant, S. V.; Shin, H.; Oliveira, J. T.; Gomes, M. 798 E.; Neves, N. M.; Khademhosseini, A.; Reis, R. L. Modified Gellan Gum 799 hydrogels with tunable physical and mechanical properties. Biomaterials 800 2010, 31 (29), 7494-502.

801 (45) Stephen, A. M.; Phillips, G. O.; Williams, P. A. Food 802 Polysaccharides and their Applications, 2nd ed.; Press, C., Ed.; Taylor \& 803 Francis Group: New York, 2006.

804 (46) Polk, A.; Amsden, B.; De Yao, K.; Peng, T.; Goosen, M. F. 805 Controlled release of albumin from chitosan-alginate microcapsules. J. 806 Pharm. Sci. 1994, 83 (2), 178-85.

807 (47) Naciri, M.; Kuystermans, D.; Al-Rubeai, M. Monitoring pH and 808 dissolved oxygen in mammalian cell culture using optical sensors. 809 Cytotechnology 2008, 57 (3), 245-50.

810 (48) Baker, L. G.; Specht, C. A.; Donlin, M. J.; Lodge, J. K. Chitosan, 811 the deacetylated form of chitin, is necessary for cell wall integrity in 812 Cryptococcus neoformans. Eukaryotic Cell 2007, 6 (5), 855-67.

813 (49) Popa, E. G.; Carvalho, P. P.; Dias, A. F.; Santos, T. C.; Santo, V. E.; 814 Marques, A. P.; Viegas, C. A.; Dias, I. R.; Gomes, M. E.; Reis, R. L. 815 Evaluation of the in vitro and in vivo biocompatibility of carrageenan816 based hydrogels. J. Biomed. Mater. Res., Part A 2014, DOI: 10.1002/ 817 jbm.a.35081.

818 (50) Popa, E.; Reis, R.; Gomes, M. Chondrogenic phenotype of 819 different cells encapsulated in kappa-carrageenan hydrogels for cartilage 820 regeneration strategies. Biotechnol. Appl. Biochem. 2012, 59 (2), 132-41. 821 (51) Fedorovich, N. E.; Haverslag, R. T.; Dhert, W. J.; Alblas, J. The 822 role of endothelial progenitor cells in prevascularized bone tissue 823 engineering: development of heterogeneous constructs. Tissue Eng., Part 824 A 2010, 16 (7), 2355-67.

825 (52) Urbich, C.; Dimmeler, S. Endothelial progenitor cells: character826 ization and role in vascular biology. Circulation Res. 2004, 95 (4), 34382753.

828 (53) Kong, H. J.; Smith, M. K.; Mooney, D. J. Designing alginate 829 hydrogels to maintain viability of immobilized cells. Biomaterials 2003, 83024 (22), 4023-9.

831 (54) Lutolf, M. P.; Gilbert, P. M.; Blau, H. M. Designing materials to 832 direct stem-cell fate. Nature 2009, 462 (7272), 433-41.

833 (55) Bryant, S. J.; Anseth, K. S. The effects of scaffold thickness on 834 tissue engineered cartilage in photocrosslinked poly(ethylene oxide) 835 hydrogels. Biomaterials 2001, 22 (6), 619-26.

836 (56) Park, J. H.; Chung, B. G.; Lee, W. G.; Kim, J.; Brigham, M. D.; 837 Shim, J.; Lee, S.; Hwang, C. M.; Durmus, N. G.; Demirci, U.; 838 Khademhosseini, A. Microporous cell-laden hydrogels for engineered 839 tissue constructs. Biotechnol. Bioeng. 2010, 106 (1), 138-48.

840 (57) Bai, Z.; Mendoza Reyes, J. M.; Montazami, R.; Hashemi, N. On841 chip development of hydrogel microfibers from round to square/ribbon 842 shape. J. Mater. Chem. A 2014, 2, 4878-4884.

843 (58) Adams, C. S.; Mansfield, K.; Perlot, R. L.; Shapiro, I. M. Matrix 844 regulation of skeletal cell apoptosis. Role of calcium and phosphate ions. 845 J. Biol. Chem. 2001, 276 (23), 20316-22.

846 (59) Murphy, C. L.; Sambanis, A. Effect of oxygen tension and alginate 847 encapsulation on restoration of the differentiated phenotype of passaged 848 chondrocytes. Tissue Eng. 2001, 7 (6), 791-803.
(60) Pirraco, R. P.; Melo-Ferreira, B.; Santos, T. C.; Frias, A. M.; 849 Marques, A. P.; Reis, R. L. Adipose stem cell-derived osteoblasts sustain 850 the functionality of endothelial progenitors from the mononuclear 851 fraction of umbilical cord blood. Acta Biomater. 2013, 9 (2), 5234-42. 852 (61) Pirraco, R. P.; Marques, A. P.; Reis, R. L. Cell interactions in bone 853 tissue engineering. J. Cell. Mol. Med. 2010, 14 (1-2), 93-102. 